\title{
Correction to: International experience in the development of patient- derived xenograft models of diffuse intrinsic pontine glioma
}

\author{
Maria Tsoli ${ }^{1}$. Han Shen ${ }^{1} \cdot$ Chelsea Mayoh $^{1} \cdot$ Laura Franshaw $^{1} \cdot$ Anahid Ehteda $^{1} \cdot$ Dannielle Upton $^{1}$. \\ Diana Carvalho ${ }^{2}$ - Maria Vinci ${ }^{2}$. Michael H. Meel ${ }^{3}$. Dannis van Vuurden ${ }^{3}$. Alexandre Plessier ${ }^{4}$. \\ David Castel $^{4} \cdot$ Rachid Drissi $^{5}$. Michael Farrell ${ }^{6}$. Jane Cryan ${ }^{6}$. Darach Crimmins ${ }^{7}$. John Caird ${ }^{7}$. Jane Pears ${ }^{8}$. \\ Stephanie Francis ${ }^{9} \cdot$ Louise E. A. Ludlow $^{10}$. Andrea Carai ${ }^{11}$. Angela Mastronuzzi ${ }^{12}$. Bing Liu ${ }^{1}$. Jordan Hansford ${ }^{10}$. \\ Nicholas G. Gottardo ${ }^{13} \cdot$ Tim Hassall $^{14} \cdot$ Maria Kirby $^{15} \cdot$ Maryam Fouladi $^{5} \cdot$ Cynthia Hawkins $^{16} \cdot$ Michelle Monje $^{17}$. \\ Jacques Grill ${ }^{4}$. Chris Jones ${ }^{2}$. Esther Hulleman ${ }^{3}$. David S. Ziegler ${ }^{1,9}$
}

Published online: 27 November 2018

(c) Springer Science+Business Media, LLC, part of Springer Nature 2018

\section{Correction to: Journal of Neuro-Oncology} https://doi.org/10.1007/s11060-018-03038-2

There are two errors and one omission in the original article. Author Upton's correct name is Dannielle Upton, author Plessier's correct name is Alexandre Plessier, author Gottardo's correct name is Nicholas G. Gottardo, author Hulleman's correct affiliation is no. 3

The original article can be found online at https://doi.org/10.1007/ s11060-018-03038-2.

David S. Ziegler

d.ziegler@unsw.edu.au

1 Children's Cancer Institute, Randwick, NSW 2031, Australia

2 Divisions of Molecular Pathology and Cancer Therapeutics, The Institute of Cancer Research, London, UK

3 Department of Pediatric Oncology, VU University Medical Center, Amsterdam, the Netherlands

4 Unite Mixte de Recherche 8203 du Centre National de la Recherche Scientifique (CNRS) et Departement de Cancerologie de l'Enfant et de l'Adolescent, Gustave Roussy et Universite Paris-Saclay, Villejuif, France

5 Brain Tumor Center, Cancer and Blood Diseases Institute, Cincinnati Children's Hospital Medical Center, 3333 Burnet Avenue, Cincinnati, OH, USA

6 Histopathology Department, Beaumont Hospital, Dublin, Ireland

7 Department of Neurosurgery, Temple Street Children's University Hospital, Dublin, Ireland

8 Our Lady's Children's Hospital, Dublin, Ireland
(VUMC, Amsterdam), and the Acknowledgements should include the following sentence: "We would like to thank Dr Angel Montero Carcaboso (Hospital Sant Joan de Deu, Barcelona, Spain) for generously supplying the HSJDDIPG007 cells."
9 Kids Cancer Centre, Sydney Children's Hospital, Randwick, NSW 2052, Australia

10 Murdoch Children's Research Institute, The Royal Children's Hospital, Parkville, VIC 3052, Australia

11 Neurosurgery Unit, Department of Neuroscience and Neurorehabilitation, Bambino Gesù Children's Hospital, Rome, Italy

12 Neuro-Oncology Unit, Department of Hemato-Oncology, Cell and Gene Therapy, Bambino Gesù Children's Hospital, Rome, Italy

13 Department of Oncology, Princess Margaret Hospital, Perth, WA, Australia

14 Lady Cilento Children's Hospital, Brisbane, Australia

15 Department of Haematology-Oncology, Women's and Children's Hospital, Adelaide, SA, Australia

16 The Arthur and Sonia Labatt Brain Tumour Research Centre, The Hospital for Sick Children, Toronto, ON, Canada

17 Stanford University and Lucile Packard Children's Hospital, Palo Alto, CA, USA 